Canadian Journal of Fisheries and Aquatic Sciences Journal canadien des sciences halieutiques et aquatiques

\title{
Including unsexed individuals in sex-specific growth models
}

\begin{tabular}{|r|l|}
\hline Journal: & Canadian Journal of Fisheries and Aquatic Sciences \\
\hline Manuscript ID & cjfas-2016-0450.R1 \\
\hline Manuscript Type: & Article \\
\hline Complete List of Authors: & $\begin{array}{l}\text { Minto, Cóilín; Galway-Mayo Institute of Technology, Marine and Freshwater } \\
\text { Research Centre } \\
\text { Hinde, John ; National University of Ireland - Galway, School of } \\
\text { Mathematics, Statistics and Applied Mathematics } \\
\text { Coelho, Rui; Centre of Marine Sciences (CCMAR). Univ. Algarve, ; } \\
\text { Portuguese Institute for the Ocean and Atmosphere (IPMA, I.P.), }\end{array}$ \\
\hline Please Select from this Special \\
Issues list if applicable:
\end{tabular}




\section{Including unsexed individuals in sex-specific growth}

$4{ }^{1}$ Corresponding author: Marine and Freshwater Research Centre (MFRC), Galway-Mayo In-

5 stitute of Technology (GMIT), Dublin Road, Galway, Ireland. E-mail: coilin.minto@gmit.ie.

6 Phone: +35391742 514. Fax: +35391758412.

$7{ }^{2}$ School of Mathematics, Statistics and Applied Mathematics, National University of Ireland,

8 Galway, Ireland. E-mail: john.hinde@ nuigalway.ie

$9{ }^{3}$ Portuguese Institute for the Ocean and Atmosphere (IPMA), Av. 5 de Outubro s/n, 8700-305

10 Olhão, Portugal. E-mail: rpcoelho@ualg.pt

$11{ }^{4}$ Centre of Marine Sciences (CCMar), Univ. Algarve, Campus de Gambelas FCT Ed. 7, 8005-

12139 Faro, Portugal.

13 
Sexually dimorphic growth models are typically estimated by fitting growth curves to individuals of known sex. Yet, macrospically ascribing sex can be difficult, particularly for immature animals. As a result, sex-specific growth curves are often fit to known-sex individuals only, omitting unclassified immature individuals occupying an important region of the age-length space. We propose an alternative whereby the sex of the unclassified individuals is treated as a missing data problem to be estimated simultaneously with the sex-specific growth models. The mixture model we develop includes the biological processes of growth and sexual dimorphism. Simulations show that where the assumed growth model holds, the method improves precision and bias of all parameters relative to the data ommission. Ability to chose the correct combination of sex-specific and sex-generic parameters is also improved. Application of the method to two shark species -where sex can be ascribed from birth- indicates improvements in the fit but also highlights the importance of the assumed model forms. The proposed method avoids discarding unclassified observations thus improving our understanding of dimorphic growth.

30 Key words: Dimorphism; EM algorithm; missing data; non-linear clustering; partial classifi31 cation. 


\section{Introduction}

Growth is a central process in the life history of an organsim (Beverton and Holt 1957; Werner 1986; Starck and Ricklefs 1998; Nentwig 2012) and describing growth is a regular task across many taxa (Lacointe 2000; Krebs and Cowan 1962; Starck and Ricklefs 1998; Jones et al. 2011). Reflecting the importance of understanding growth, modelling growth has a long and productive history in ecology (Robertson 1923; Brody 1926; von Bertalanffy 1938). A commonly applied parametric functional form for the relationship between length and age of given life history stages is the von Bertalanffy model (von Bertalanffy 1938; Chen et al. 1992). Early methods to fit this non-linear model used the linear differenced relationship between mean lengths separated by a unit of age (Ford 1933; Walford 1946). Maximum likelihood fitting under the assumption of normally distributed errors was introduced by Kimura (1980). A sophisticated range of fitting methods now exists, including: non-normal error distributions, more stable re-parameterizations (Gallucci and Quinn 1979; Schnute 1981; Francis 1988), hierarchical methods (Helser and Lai 2004; Brunel and Dickey-Collas 2010; Weisberg et al. 2010), and error-in-variables approaches (Cope and Punt 2007).

Sexually dimorphic growth occurs in many taxa (Fairbairn et al. 2007). Differential selection pressures, mortality schedules and the degree of reproductive investment affect the ultimate differences in size (Roff 1982; Parker 2006). Where it occurs, sexually dimorphic growth has important implications for management. Natural mortality can be size-dependent (Pauly 1980). Harvesting methods are also often size-based, such that the probability of removal changes with size, e.g., small fish might escape through the mesh whereas larger fish are too big to escape (Myers and Hoenig 1997). Where differences exist in the growth of the sexes, selective sizebased removal will enact differential mortality between the sexes (Kendall and Quinn 2012). To understand and mitigate for size-selective removals, it is essential that sex-specific growth characteristics are well described.

On first inspection, fitting sex-specific growth models to length-at-age data requires that the sex of an individual is known. For maturing and mature animals, sex can often be assigned visually from primary or secondary sexual characteristics, but immature fish, amphibians, insects and reptiles often require histological methods to distinguish between the sexes (e.g., Vitale et al. 
2006). Histological methods are often not feasible for large-scale continuous sampling programs. As a result, many sex-specific growth datasets contains the sex-designations: Female, Male and Unclassified (FMU).

Extant approaches to fitting sex-specific growth curves to FMU data proceed by first removing unsexed (immature or otherwise unclassified) individuals and then fitting sex-specific curves to the remaining known-sex individuals (e.g., using the methods of Kimura (1980)). Sex-specific growth models of this type are thus fit to a subset of the observed age-length data; we term these FMU fits. FMU fits introduce an extrapolation of the model over an omitted space (typically young immature animals) that may be very informative for certain parameters of the model, particularly the growth rate and intercept. As an extreme illustration, consider a species with determinate growth (somatic growth ceases post-maturation) with all individuals undergoing maturation at a given age (when the sex can be determined). Known-sex lengths will be constant over age and therefore only informative for sex-specific asymptotic size. Without additional assumptions, sex-specific growth rates and intercepts will be confounded. In reality, many species such as many molluscs, fish and reptiles have indeterminate growth (growth continues post-maturation) but we contend that fitting sex-specific growth curves to FMU data in the extant fashion amounts to extrapolation, the severity of which will depend on: ability to determine sex; duration of the immature stage; degree of post-maturation growth; and how protracted the maturation process is across individuals.

Instead of omitting unclassified individuals when fitting sex-specific growth models to FMU data, we propose that the sex of the unclassified individuals be treated as a missing data problem to be estimated simultaneously with the growth curves. We develop a mixture model for this purpose where few restrictions are made on the form of the growth curves or on the between- or within-individual variability but we do focus on the von Bertalanffy model, as a commonly applied functional form (Pardo et al. 2013).

Our objectives are to: (1) develop a mixture model for including unclassified individuals in sex-specific growth curves; (2) develop an accompanying expectation-maximisation (EM) fitting algorithm (Dempster et al. 1977); (3) test the performance of the method relative to the extant FMU approach via simulation; and (4) fit to two species of shark where known sexes of 
immature and mature individuals provide a useful real data test of performance.

\section{Materials and Methods}

93 We first motivate the unsexed individuals as a missing data problem, then propose a mixture 94 model solution with an accompanying EM-algorithm estimation routine; simulation tests and 95 real data applications follow.

\section{A partially classified two-component mixture model}

97 The observed data consist of a sample of $i=1, \ldots n$ individuals with recorded variables: length $98 l_{i}$, age $a_{i}$, and observed sex $s_{o b s, i}$ where $s_{o b s, i} \in\{F, M, U\}$ (Female, Male, Unclassified). For 99 the overall population, following the notation of McLachlan and Peel (2000), we define a two100 component (female $F$ and male $M$ ) mixture model with a length probability density function 101 of:

$$
f(\boldsymbol{l} \mid \boldsymbol{a}, \Psi)=\pi_{F} f_{F}\left(\boldsymbol{l} \mid \boldsymbol{a}, \boldsymbol{\theta}_{\boldsymbol{F}}\right)+\pi_{M} f_{M}\left(\boldsymbol{l} \mid \boldsymbol{a}, \boldsymbol{\theta}_{\boldsymbol{M}}\right),
$$

where: $\boldsymbol{\Psi}=\left\{\pi_{F}, \boldsymbol{\theta}_{\boldsymbol{F}}, \boldsymbol{\theta}_{\boldsymbol{M}}\right\}$ is a vector of all free parameters; $\pi_{F}$ is the mixing proportion, 103 which is the overall probability that the sex is female, $\pi_{F}=P(S=F)$, and the complement $104 \pi_{M}=1-\pi_{F}$ is the overall probability that the sex is male; $f_{S}$ is the sex-specific (female 105 or male) component density, e.g., lognormal for a single observation (presented without bias 106 correction):

$$
f_{S}\left(l_{i} \mid a_{i}, \boldsymbol{\theta}_{\boldsymbol{S}}\right)=\frac{1}{l_{i} \sigma_{S} \sqrt{2 \pi}} \exp \left(-\frac{\left(\ln \left(l_{i}\right)-\ln \left(g\left(a_{i}, \boldsymbol{\Omega}_{\boldsymbol{S}}\right)\right)\right)^{2}}{2 \sigma_{S}^{2}}\right)
$$

107 where $\boldsymbol{\theta}_{\boldsymbol{S}}=\left\{\sigma_{S}, \boldsymbol{\Omega}_{\boldsymbol{S}}\right\}$ is the sex-specific density parameter vector; $g\left(a_{i}, \boldsymbol{\Omega}_{\boldsymbol{S}}\right)$ is the sex-specific 108 growth function, e.g., von Bertalanffy

$$
g\left(a_{i}, \boldsymbol{\Omega}_{\boldsymbol{S}}\right)=L_{\infty, S}\left(1-e^{-K_{S}\left(a_{i}-t_{0, S}\right)}\right),
$$


109 where $\Omega_{S}=\left\{L_{\infty, S}, K_{S}, t_{0, S}\right\}$ is the sex-specific growth curve parameter vector consisting here

110 of the mean asymptotic length, Brody growth rate (rate at which asymptote is approached) and

111 age at length zero, respectively. Alternative forms for $g$ include other asymptotic, segmented,

112 and additive curves.

113 To estimate the parameters of the mixture model, we introduce the partially observed indicator

114 variable $Z$ that denotes the true sex of observation $i$

$$
z_{i}= \begin{cases}1, & \text { if observation } i \text { is female, } s_{i}=F \\ 0, & \text { if observation } i \text { is male, } s_{i}=M\end{cases}
$$

115 Letting $i=1, \ldots, m$ index the classified individuals for which the sex is known, we propose 116 that for the remaining $j=m+1, \ldots, n$ individuals, $z_{j}$ can be treated as missing data to be

117 imputed from the posterior probability of an unclassified observation being female. Next, we 118 introduce how classified and unclassified individuals are treated within the estimation.

\section{Classified individuals}

120 For each $i$, the true value of $z_{i} \in\{0,1\}$ and is known. The conditional density of the classified

121 observation can therefore be written

$$
f\left(l_{i} \mid a_{i}, z_{i}, \boldsymbol{\Psi}\right)=f_{F}\left(l_{i} \mid a_{i}, \boldsymbol{\theta}_{\boldsymbol{F}}\right)^{z_{i}} f_{M}\left(l_{i} \mid a_{i}, \boldsymbol{\theta}_{\boldsymbol{M}}\right)^{1-z_{i}}
$$

122 and the density of the group (female or male) membership

$$
f\left(z_{i} \mid \Psi\right)=\pi_{F}^{z_{i}} \pi_{M}^{1-z_{i}}
$$

123 Assuming independence, the joint density of the classified observation and the group member-

124 ship can thus be written

$$
f\left(l_{i}, z_{i} \mid a_{i}, \boldsymbol{\Psi}\right)=\left[\pi_{F} f_{F}\left(l_{i} \mid a_{i}, \boldsymbol{\theta}_{\boldsymbol{F}}\right)\right]^{z_{i}}\left[\pi_{M} f_{M}\left(l_{i} \mid a_{i}, \boldsymbol{\theta}_{\boldsymbol{M}}\right)\right]^{1-z_{i}}
$$


125 The joint density of all the classified observations is

$$
f(\boldsymbol{l}, \boldsymbol{z} \mid \boldsymbol{a}, \boldsymbol{\Psi})=\prod_{i=1}^{m}\left[\pi_{F} f_{F}\left(l_{i} \mid a_{i}, \boldsymbol{\theta}_{\boldsymbol{F}}\right)\right]^{z_{i}}\left[\pi_{M} f_{M}\left(l_{i} \mid a_{i}, \boldsymbol{\theta}_{\boldsymbol{M}}\right)\right]^{1-z_{i}}
$$

131 Equation (1), as:

$$
f\left(l_{j} \mid a_{j}, \boldsymbol{\Psi}\right)=\pi_{F} f_{F}\left(l_{j} \mid a_{j}, \boldsymbol{\theta}_{\boldsymbol{F}}\right)+\pi_{M} f_{M}\left(l_{j} \mid a_{j}, \boldsymbol{\theta}_{\boldsymbol{M}}\right) .
$$

132 For each $j$, the true value of $z_{j} \in\{0,1\}$ but is now unknown. Similar to Dean et al. (2006) and 133 McLachlan and Krishnan (2008), a key quantity is the expected value of the group membership 134 indicator for unclassified individuals given the observed data and a given set of parameters 135 (denoted by an asterisk)

$$
\mathrm{E}\left(Z_{j} \mid a_{j}, l_{j}, \Psi^{*}\right)=\operatorname{Pr}\left(Z_{j}=1 \mid a_{j}, l_{j}, \Psi^{*}\right) .
$$

136 This pivotal probability is available via Bayes' theorem (Bayes 1764), as:

$$
\begin{aligned}
\operatorname{Pr}\left(Z_{j}=1 \mid a_{j}, l_{j}, \boldsymbol{\Psi}^{*}\right) & =\frac{\operatorname{Pr}\left(Z_{j}=1\right) \operatorname{Pr}\left(l_{j} \mid z_{j}=1, a_{j}, \boldsymbol{\Psi}^{*}\right)}{\operatorname{Pr}\left(l_{j} \mid a_{j}, \boldsymbol{\Psi}^{*}\right)}, \\
& =\frac{\pi_{F}^{*} f_{F}\left(l_{j} \mid a_{j}, \boldsymbol{\theta}_{\boldsymbol{F}}^{*}\right)}{\pi_{F}^{*} f_{F}\left(l_{j} \mid a_{j}, \boldsymbol{\theta}_{\boldsymbol{F}}^{*}\right)+\pi_{M}^{*} f_{M}\left(l_{j} \mid a_{j}, \boldsymbol{\theta}_{\boldsymbol{M}}^{*}\right)} .
\end{aligned}
$$

137 The estimated component membership probabilities are denoted by $z_{j}^{*}$. Equation (12) thus 138 provides a means for assigning the probability of group (i.e., female or male) membership to 139 unclassified individuals for a given growth model and set of parameters. We can thus proceed, 140 as for classified individuals, to an expected value for the log-likelihood, conditional on the 


$$
\mathrm{E}\left[\ln L\left(\Psi \mid \boldsymbol{l}_{\boldsymbol{j}}, \boldsymbol{z}_{j}^{*}, \boldsymbol{a}_{\boldsymbol{j}}\right)\right]=\sum_{j=m+1}^{n} z_{j}^{*} \ln \left(\pi_{F} f_{F}\left(l_{j} \mid a_{j}, \boldsymbol{\theta}_{\boldsymbol{F}}\right)\right)+\left(1-z_{j}^{*}\right) \ln \left(\pi_{M} f_{M}\left(l_{j} \mid a_{j}, \boldsymbol{\theta}_{\boldsymbol{M}}\right)\right)
$$

142 As an aside, the simplicity here is due to the log-likelihood based on a representation, as in 143 Equation (8), that is linear in $z_{j}^{*}$. Combining the classified and unclassified individuals, the expected complete data log-likelihood of all observations is then given by

$$
\begin{aligned}
& \mathrm{E}[\ln L(\boldsymbol{\Psi} \mid \boldsymbol{l}, \boldsymbol{z}, \boldsymbol{a})]= \\
& \sum_{i=1}^{m}\left[z_{i} \ln \left(\pi_{F} f_{F}\left(l_{i} \mid a_{i}, \boldsymbol{\theta}_{\boldsymbol{F}}\right)\right)+\left(1-z_{i}\right) \ln \left(\pi_{M} f_{M}\left(l_{i} \mid a_{i}, \boldsymbol{\theta}_{\boldsymbol{M}}\right)\right)\right]+ \\
& \sum_{j=m+1}^{n}\left[z_{j}^{*} \ln \left(\pi_{F} f_{F}\left(l_{j} \mid a_{j}, \boldsymbol{\theta}_{\boldsymbol{F}}\right)\right)+\left(1-z_{j}^{*}\right) \ln \left(\pi_{M} f_{M}\left(l_{j} \mid a_{j}, \boldsymbol{\theta}_{\boldsymbol{M}}\right)\right)\right]
\end{aligned}
$$

\section{EM algorithm}

146 Estimation of the expected log-likelihood (Equation 14) is carried out by the following steps:

1. E-step

Starting with a given set of parameters $\Psi^{(0)}=\left\{\boldsymbol{\theta}^{(0)}, \pi_{F}^{(0)}\right\}$, calculate the expected value of the $\log$-likelihood (Equation 14), which amounts to finding $z_{j}^{*}$ in Equation (12).

2. M-step

a. Maximise the expected value of the complete data log-likelihood with respect to the growth parameters

$$
\boldsymbol{\theta}^{(1)}=\underset{\boldsymbol{\theta}}{\operatorname{argmax}} \mathrm{E}\left[\ln L\left(\boldsymbol{\theta} \mid \boldsymbol{l}, \boldsymbol{z}^{(0)}, \boldsymbol{a}, \pi_{F}^{(0)}\right)\right] .
$$

Non-linear growth models require numerical optimisation for this step.

b. Update the unconditional probability of being female (sex ratio), if not assumed fixed, via

$$
\pi_{F}^{1}=\frac{\sum_{i=1}^{m} z_{i}+\sum_{j=m+1}^{n} z_{j}^{*(0)}}{n}
$$


which assumes the same overall sex ratio for the unclassified and classified; but this can be relaxed to classified or unclassified only, fixed at a given value, or modelled with covariates where data allow.

3. Replace $\boldsymbol{\Psi}^{(0)}$ with $\boldsymbol{\Psi}^{(1)}=\left\{\boldsymbol{\theta}^{(1)}, \pi_{F}^{(1)}\right\}$ and repeat steps 1 and 2 until convergence, as assessed by a stopping criteria based on a relative change in the observed log-likelihood. The observed log-likelihood is given by

$$
\begin{array}{r}
\ln L\left(\Psi^{*} \mid \boldsymbol{l}, \boldsymbol{z}, \boldsymbol{a}\right)=\sum_{i=1}^{m}\left(z_{i} \ln \left(\pi_{F}^{*} f_{F}\left(l_{i} \mid a_{i}, \boldsymbol{\theta}_{\boldsymbol{F}}^{*}\right)\right)+\left(1-z_{i}\right) \ln \left(\pi_{M}^{*} f_{M}\left(l_{i} \mid a_{i}, \boldsymbol{\theta}_{\boldsymbol{M}}^{*}\right)\right)\right)+ \\
\sum_{j=m+1}^{n} \ln \left(\pi_{F}^{*} f_{F}\left(l_{j} \mid a_{j}, \boldsymbol{\theta}_{\boldsymbol{F}}^{*}\right)+\pi_{M}^{*} f_{M}\left(l_{j} \mid a_{j}, \boldsymbol{\theta}_{\boldsymbol{M}}^{*}\right)\right) .
\end{array}
$$

Note that it may be possible to maximise this partially classified observed likelihood directly without the EM algorithm, but direct maximisation does not in general perform well, except in the vicinity of the maximum or via a grid search.

\section{Parameter inference and standard errors}

In the same manner as Kimura (1980), parameters may be bound or free across the sexes.

We fit all combinations of bound and free parameters and choose the best fitting model by Bayesian Information Criterion (BIC using the: observed data log-likelihood (Equation 17); log of the number of observations; and number of parameters of the component distribution and the mixing proportion). A preliminary investigation of the performance of the Akaike Information Criterion showed it to select overly complex models compared to BIC. Various options are available to approximate the standard errors of the parameter estimates from an EM algorithm (McLachlan and Peel 2000). A key difficulty is obtaining the observed information matrix. The method we implement is to fit the model to convergence via the EM algorithm as above and then optimise the partially-classified mixture log-likelihood (Equation 17) directly, including the mixing proportion. The EM algorithm finds the maximum likelihood which would be difficult to do via direct optimisation. Once at the maximum, direct optimisation is relatively straightforward and can be used to estimate the curvature of the observed log-likelihood and 
hence provide standard errors.

180

18

182

183

184

185

186

187

188

189

190

191

192

202 The performance of the methods on the simulated data was appraised using: 1) parameter root203 median-square error (RMSE); 2) classification rates (based on maximum a posteriori / binary 204 classification); 3) ability to chose the correct model form via BIC; and 4) visually via boxplots 205 of the relative error $\left(\left(\hat{\theta}-\theta_{\text {true }}\right) / \theta_{\text {true }}\right)$ of the parameter estimates. 
207 Elasmobranchs (sharks and rays), represent a useful taxon to test the performance of the method 208 given that the sex can be distinguished from birth, owing to two extensions of the pelvic fins 209 (claspers) on the males. We use two datasets on deepwater lantern shark species: velvet belly, 210 Etmopterus spinax, and smooth lanternshark, Etmopterus pusillus, which are found off the 211 south and southwestern coasts of Portugal (Coelho et al. 2005). The datasets consist of 733 ( $E$. 212 spinax) and 518 (E. spinax) age-length readings. The data are cross-sectional in that there is a 213 single data point per individual. Females represented approximately $60 \%$ (E. spinax) and $43 \%$ 214 (E. pusillus) of the samples, respectively. High proportions of the individuals were classified 215 as immature (E. spinax: 60\%; E. spinax: 77\%) based on macroscopic investigation of the 216 reproductive organs (Coelho and Erzini 2007, 2008).

217 To test the performance of the method, we compare sex-specific von Bertalanffy fits from: (1) 218 all individuals, using mature and immature sex determinations; (2) only the mature animals 219 to mimic the FMU situation where sex cannot be distinguished prior to the onset of maturation; 220 and (3) all individuals with the sex of the immature animals treated as an unknown classification 221 problem and fit via the mixture model developed here. A grid of plausible starting values was 222 used to initialise fitting for each method.

\section{Software}

224 We developed the R package lhmixr (life history mixture models in $R$ : available on CRAN or at 225 https://github.com/mintoc/lhmixr/) to fit sex-specific growth models with missing classifica226 tions. von Bertalanffy models are implemented with analytical gradients for all parameters in 227 the complete-data log-likelihood and a generic binding formulation to allow parameters to be 228 bound equal or free between the sexes. Normal and log-normal error distribution assumptions 229 are included. Both real datasets are included and documented in the lhmixr package. 


\section{Results}

\section{Simulation performance}

232 Example simulation fits show that, where fish can be classified early (Figure 2(A)), the FMU 233 approach can perform well in recovering the full data curve, though with decreased precision 234 (Table 1, Figure 3). In contrast, for late maturing animals the example FMU fit performed 235 poorly for males and females (Figure 2(B)) and in general had increased bias and decreased 236 precision for the growth rate and intercept terms, particularly for strongly asymptotic growth 237 curves (Table 1, Figure 3 (C, E)).

240 Overall, as judged by RMSE, the MIX model (mixture model developed here) outperforms the FMU approach for all parameters and both curve typologies (Table 1). The differences in

242 RMSE are most pronounced in the asymptotic fits where the MIX-estimated intercept $t_{0}$ often 243 displays an order of magnititude difference in RMSE over the FMU fits (Table 1). For early ma244 turing animals with linear growth, the RMSE is reduced by half or more using the MIX method 245 relative to the FMU approach (Table 1). The MIX method consistently had RMSE slightly 246 larger than the full knowledge fit, reflecting the additional uncertainty when classification is 247 needed (Table 1).

[Figure 2 about here.]

249 All methods had similar ability to detect sex-specific difference in the asymptotic size, irre250 spective of curve typology or maturation timing (Table $2, L_{\infty, s}$ scenarios). Ability to detect 251 differences in the growth rate is considerably reduced in the FMU fits compared to the full 252 data fits, particularly for late maturing animals with more asymptotic growth where the correct 253 model was identified in only $14 \%$ of simulations (Table 2, asymptotic growth form and late 254 maturing FMU $K_{s}$ scenario). This was improved to $46 \%$ chosen correct using the MIX model. 255 The performance is not as poor for the more linear growth form (33\% FMU chosen correctly), 256 though the MIX model still improved the performance (69\% chosen correctly). When $t_{0}$ dif257 fers by sex and the growth curve typology is asymptotic, the FMU approach performed poorly 
258 in choosing the correct model (7\% and $2 \%$ for early and late maturing animals, respectively)

259 (Table 2, FMU $t_{0, s}$ scenarios); this is somewhat improved for early maturing animals using the 260 MIX model (36\%) but is low for the late maturing animals (24\%) (Table 2, MIX $t_{0, s}$ scenar261 ios). With asymptotic growth and where $t_{0}$ or $K$ differ by sex and the FMU approach is used, 262 the chosen model is often that of no difference between the sexes (Table 2, FMU None row). 263 Ommitted models from Table (2) often included the correct model but with residual standard 264 deviation differing by sex (e.g., where only $L_{\infty}$ differs by sex, a model with $L_{\infty, s}$ and $\sigma_{s}$ is the 265 most commonly chosen model of those not shown).

267 Working with the best fitting model per simulation, the classification rates where typically 268 lower when using the FMU model; whereas the MIX model recovered similar but consistently 269 lower classification rates to the FULL data scenario (Table 3). Relative to the FMU approach, 270 large gains in the classification rates are seen where growth is asymptotic (Table 3, third row). 271 As expected, classification rates are poor across all methods where no true differences exist 272 among the sexes (Table 3, "None" columns).

274 Reflecting the RMSE results, differences in the relative error across methods are least for the $275 L_{\infty}$ parameter, reflecting the predominant importance of mature animals for estimating that 276 parameter (Figure 3). In many cases the relative error of the MIX model is comparable to that 277 of the full data scenario for these simulations (Figure 3).

\section{Real data performance}

280 The two elasmobranch species investigated spend a relatively long period of their observed 281 lifespan in the immature phase in addition to a relatively large size at maturation (Figure 4). 282 As a result the best fitting FMU $t_{0}$ estimates for female and male E. spinax and E. pusil283 lus are considerably more negative than when the full data are used (Table 4, sixth column). 
284 FMU-estimated intercepts also had considerably decreased precision with no evidence of a 285 sex-specific difference. These biases and lack of precision simply reflect that the FMU model excludes those immature animals and thus only fits to the mature animals. In contrast, the MIX approach results in more comparable estimates to the full data scenario.

Estimates of $L_{\infty}$ are similar across methods and both sexes for E. pusillus. The FMU and mixture models estimate a lower asymptotic size for female E. spinax (Figure 4(C,E)) most likely 0 because they are more influenced by the larger known sex females, which are fit poorly in the full data scenario (Figure 4(A)). The E. spinax male asymptotic size is estimated as lower in the FMU fit compared to the full data fit; whereas the mixture model estimated a larger and more uncertain male asymptotic size (Table 4, row 6).

4 The female E. pusillus mixture model estimated growth rate is faster than the full data scenario and results in a higher curve (Figure 4(F)). As a result many of the smaller fish for a given age are classified as male, whereas this is not true from the full data, where there is a mixture of males and females. This may arise from the choice of functional form in that there appears to be a broadly similar growth pattern for until older ages where the male growth slows considerably for both spacies(see Discussion). As the FMU approach has smaller males and the immature animals fall under both curves, the predicted classification is unity for males but very poor for females (Table 4). The mixture model improves on this but goes to a solution that separates the sexes more than is apparent in the true data (Figure 4). Overall the classification rates from all models, including the full data scenario, are poor across both sexes owing to a lack of clear separation.

[Figure 4 about here.]

\section{Discussion}

8 Discarding unclassified data degrades the precision and accuracy of fitted sex-specific growth 909 models. Here, we have proposed a redress that simultaneously attempts to classify unclassified 10 individuals while estimating parameters of a growth curve of interest. To our knowledge this 
311 is the first recognition of the potential to couple classification and life history model parameter 312 estimation in this manner.

\section{Performance}

314 Where the fitting assumptions match the data-generating assumptions, as in our simulations,

315 the mixture model offers general improvements on the estimation of sex-specific growth curves

316 from partially-classified data (Table 1, Figure 3). Since a lognormal distribution and the von

317 Bertalanffy model are the most common assumptions for these data we think that the method

318 should also improve fits to real datasets.

319 Where animals can be classified at early ages, the FMU approach may provide good estimates

320 of the parameters of the growth curve (Figure 2). This does have the cost of reduced precision

321 (Table 1, Figure 3), which will decrease the ability to correctly choose differences between

322 the sexes (Table 2). In reality, the impact of the ommission of unclassified individuals will

323 vary by population depending on how early immature animals can be classified. Even where

324 classification can occur at a relatively young age, maturity is often dependent on reaching a

325 threshold size (Stearns and Koella 1986). Within an early age-group, larger fish may mature

326 first and hence be distinguished earlier, which may further bias estimates of FMU-derived sex-

327 specific growth compared to when all individuals are included, as proposed here.

328 Other solutions in the FMU case may include fixing the intercept at a certain value. Though this

329 may seem a simpler solution, it is not recommended given the resulting bias in the growth rate

330 for single fits (Pardo et al. 2013). In addition, for sex-specific fits, it is difficult to envisage how

331 fixing the intercept term would not bias the other sex-specific parameters where differences 332 exist.

333 Lantern shark datasets investigated demonstrated some extreme challenges to fitting with the 334 FMU approach (Figures 4). In reality, it may be possible to ascribe sex for some individuals 335 prior to maturity but these fits serve to emphasise some of the issues, as discussed below. 
A primary concern with the von Bertalanffy model is that the functional form of the growth curve differs between the immature and mature phases. Such valid concerns have given rise to alternative mechanistic growth models such as the biphasic growth model of Roff (1983) where somatic growth is linear to the onset of maturity and thereafter a decreasing function of the gonadosomatic index. The von Bertalanffy model theoretically describes only post-maturation growth (Ricker 1975; Lester et al. 2004). We stress, however, that the form of the growth model ( $g$ in Equation 3) we use for our derivation or implementation of the algorithm is not restricted and our use of the von Bertalanffy form only reflects its common application. However, hockey-stick implementations of sex-specific Roff biphasic growth models could be very difficult to fit to asymptotic datasets containing unclassified immature animals. The mixture model developed here may contribute in this regard. Overall, it is important to recognise that the method classifies on the basis of the assumed model, so a functional form mis-match may result in poor classification (e.g., E. pusillus, Figure 4). It is difficult to guard against this, other than by comparing with more local functional forms.

1 The error distribution assumption will affect the classification - we therefore recommend careful consideration of the error-distribution and the mean-variance relationship thus implied. In the lhmixr package we provide two error distributions (normal and lognormal) but envisage other density forms could be useful in particular applications (e.g., gamma, heteroscedastic formulations). We envisage many ways of improving and highlight the E. pusillus fits (Figure 4) as a caution of how the performance may be critiqued in given applications.

357 Of additional concern is the assumption that the sex of the individual is fixed. Fish and other taxon display a great diversity of sexual life histories (Warner 2012), including sequential hermaphroditism, where the sex of the animal can change. On first inspection, classifications to such families as Labrids or gobies, for what may be a non-constant trait, could be a less than sensible approach but again we propose a possible development via the inclusion of relevant covariates for the mixing proportions (Equation 16).

363 In terms of model choice and parameter inference, it is known that the regularity conditions of the asymptotic distribution of the deviance break down when comparing mixture models with 
differing numbers of components (McLachlan and Peel 2000). The same is true of AIC and $\mathrm{BIC}$, though BIC has been shown to perform well in some situations for identifying the number of components (Wang et al. 1996). We recommend first using BIC to compare a fully saturated two component model (all parameters sex-specific) with a single component model, where all parameters are the same across sexes. Once the number of components is decided, and interest lies in parameter significance, nested models within that number of components can be tested using likelihood ratio tests with a chi-squared distribution. Overall, we propose that the asymptotic distribution of the deviance in this partially classified mixture model requires further work.

\section{Management implications}

Given that yields and mortality schedules are directly impacted by growth parameters, having reliable estimates of sex-specific growth parameters is paramount for management of dimorphic species. While the mixture model offers general improvements over the unclassified ommission case (Figure 3), we envisage the method assisting most where growth is more strongly asymptotic after maturation (Figure 2). Where faster growing individuals mature earlier and are therefore classifiable earlier, positive biases may exist in previously estimated sex-specific growth curves. This would imply faster growth than when all the data are used. In this setting the method could contribute to management by using all the data to estimate the relevant growth parameters and quantify uncertainty around the sex-specific growth curves. These curves can then be used, for example, in age-length based population assessment models (e.g., Methot 2000; Frøysa et al. 2002).

\section{Future developments}

While the approach developed here focusses on partially classified datasets, the mixture model could also be applied to datasets where no sexed animals are recorded, as in common applications to fully unclassified datasets (McLachlan and Peel 2000).

1 Extending the method longitudinally would allow for the life histories of unclassified individ- 
392 uals to be understood in the context of sex-specific differences. Longitudinal features could 393 be built in by modelling the correlation structure of the errors (e.g., using generalized least 394 squares, estimating equations or random effects (Diggle et al. 2003)).

395 Other sex-specific life history relationships may also be amenable to the methods developed 396 here. We are currently developing a similar approach for the treatment of maturity. Perceivably 397 these could be coupled into a multivariate partially classified mixture model, where it is explic398 itly recognised that animals develop along multiple coupled processes.

399 The estimation time is short (seconds) and lhmixr von Bertalanffy complete data log-likelihoods 400 are also accompanied by their analytical gradients to provide more stable fitting. The EM al401 gorithm can, however, be comparatively slow in terms of the number of iterations taken to convergance. This could be improved by implementing the loops in a compiled language (e.g., $\mathrm{C}++$ ). It would also be of particular interest to start the model at the M-step (e.g., assuming an equal probability of 0.5 for unclassified individuals) and test performance compared to starting at the E-step with a grid of starting values (as done here) or starting at the FMU solution. Bayesian estimation of finite mixture models is an active area of development (FrühwirthSchnatter 2006), which would assist straightforward estimation of parameter uncertainty using MCMC, for example, but with associated chain convergence and runtime overheads.

Despite the long history of application of mixture models to ecological problems (Pearson 1895; Bhattacharya 1967; Macdonald and Pitcher 1979) and recent applications (Thorson et al. 2011; Cosgrove et al. 2014), we note in closing that the EM algorithm is at present a con412 siderably under-recognised algorithm in ecological modelling. The EM algorithm contributes 413 to areas as diverse as state space modelling (Shumway and Stoffer 1982), structural equa414 tions (Ullman and Bentler 2003), hidden Markov systems (McLachlan and Krishnan 2008), 415 all of which are important topics in modern ecological modelling, yet other routines (MCMC, 416 Laplace, quadrature approximations to the marginal likelihood) are more commonly used when 417 estimating complex ecological models (e.g., Bolker et al. 2013; Kristensen et al. 2015). While 418 recognising the central importance of these methods, we contend that for many users there is a 419 lack of understanding as to how the methods work. In contrast, the steps of the EM algorithm 420 are transparent when estimating unobserved constructs. We believe that the EM algorithm de- 
421

veloped here demonstrates a small amount of the potential of this family of algorithms, which deserve more recognition in modern ecological modelling.

\section{Acknowledgements}

We are grateful to Dr Guillaume Bal, Marine Institute, Ireland for valuable feedback. Two anonymous reviewers are thanked for greatly improving the manuscript. We thank John Corcoran for initial discussions on sex-specific growth models for perch that instigated development of the method. CM thanks Dr Conor Nolan for encouraging insights on animal growth.

\section{References}

Bayes, T. 1764. An essay toward solving a problem in the doctrine of chances. Philosophical Transactions of the Royal Society of London 53: 370-418.

Beverton, R.J.H. and Holt, S.J. 1957. On the Dynamics of Exploited Fish Populations. Fisheries Investigations Series 2, Vol. 19. Ministry of Agriculture Fisheries and Food, London, UK.

Bhattacharya, C.G. 1967. A simple method of resolution of a distribution into Gaussian components. Biometrics 23: 115-135.

Bolker, B.M., Gardner, B., Maunder, M., Berg, C.W., Brooks, M., Comita, L., Crone, E., Cubaynes, S., Davies, T., Valpine, P. et al. 2013. Strategies for fitting nonlinear ecological models in r, ad model builder, and bugs. Methods in Ecology and Evolution 4(6): 501-512.

Brody, S. 1926. Time relations of growth: I. Genetic growth constants of animals. The Journal of General Physiology 8(3): 233-251.

Brunel, T. and Dickey-Collas, M. 2010. Effects of temperature and population density on von bertalanffy growth parameters in atlantic herring: a macro-ecological analysis. Marine Ecology Progress Series 405: 15-28.

Chen, Y., Jackson, D.A., and Harvey, H.H. 1992. A comparison of von bertalanffy and polynomial functions in modelling fish growth data. Canadian Journal of Fisheries and Aquatic Sciences 49(6): 1228-1235. 
Coelho, R. and Erzini, K. 2007. Population parameters of the smooth lantern shark, Etmopterus pusillus, in southern portugal (NE Atlantic). Fisheries Research 86(1): 42-57.

Coelho, R. and Erzini, K. 2008. Life history of a wide-ranging deepwater lantern shark in the north-east Atlantic, Etmopterus spinax (chondrichthyes: Etmopteridae), with implications for conservation. Journal of Fish Biology 73: 1419-1443.

Coelho, R., Erzini, K., Bentes, L., Correia, C., Lino, P.G., Monteiro, P., Ribeiro, J., and Gonçalves, J.M. 2005. Semi-pelagic longline and trammel net elasmobranch catches in southern portugal: catch composition, catch rates and discards. Journal of Northwest Atlantic Fishery Science 35: 531-537.

Cope, J. and Punt, A. 2007. Admitting ageing error when fitting growth curves: an example using the von Bertalanffy growth function with random effects. Canadian Journal of Fisheries and Aquatic Sciences 64(2): 205-218.

Cosgrove, R., Sheridan, M., Minto, C., and Officer, R. 2014. Application of finite mixture models to catch rate standardization better represents data distribution and fleet behavior. Fisheries Research 153: 83-88.

Dean, N., Murphy, T.B., and Downey, G. 2006. Using unlabelled data to update classication rules with applications in food authenticity studies. Journal of the Royal Statistical Society. Series C 55(1): 1-14.

Dempster, A., Laird, N., and Rubin, D. 1977. Maximum likelihood from incomplete data via the em algorithm. Journal of the Royal Statistical Society. Series B (Methodological) 39: $1-38$.

Diggle, P., Heagerty, P., Liang, K., and Zeger, S. 2003. Analysis of Longitudinal Data. Oxford University Press, Oxford, U.K.

Fairbairn, D.J., Blanckenhorn, W.U., and Székely, T. 2007. Sex, size, and gender roles: evolutionary studies of sexual size dimorphism. Oxford University Press Oxford. 
Ford, E. 1933. An account of the herring investigations conducted at Plymouth during the years from 1924 to 1933. Journal of the Marine Biological Association of the United Kingdom 19(01): 305-384.

Francis, R.I.C.C. 1988. Are growth parameters estimated from tagging and age-length data comparable? Canadian Journal of Fisheries and Aquatic Sciences 45(6): 936-942.

Frøysa, K.G., Bogstad, B., and Skagen, D.W. 2002. Fleksibestłn age-length structured fish stock assessment model. Fisheries Research 55(1): 87-101.

Frühwirth-Schnatter, S. 2006. Finite mixture and Markov switching models. Springer Science \& Business Media.

Gallucci, V. and Quinn, II, T.J. 1979. Reparameterizing, fitting, and testing a simple growth model. Transactions of the American Fisheries Society 108(1): 14-25.

Helser, T.E. and Lai, H.L. 2004. A bayesian hierarchical meta-analysis of fish growth: with an example for north american largemouth bass, Micropterus salmoides. Ecological Modelling 178(3): 399-416.

Jones, T.T., Hastings, M.D., Bostrom, B.L., Pauly, D., and Jones, D.R. 2011. Growth of captive leatherback turtles, Dermochelys coriacea, with inferences on growth in the wild: Implications for population decline and recovery. Journal of Experimental Marine Biology and Ecology 399(1): $84-92$.

Kendall, N.W. and Quinn, T.P. 2012. Size-selective fishing affects sex ratios and the opportunity for sexual selection in alaskan sockeye salmon Oncorhynchus nerka. Oikos pp. DOI: 10.1111/j.1600-0706.2012.20319.x.

Kimura, D. 1980. Likelihood methods for the von Bertalanffy growth curve. Fish. Bull 77(4): $765-776$.

Krebs, C. and Cowan, I.M. 1962. Growth studies of reindeer fawns. Canadian Journal of Zoology 40(5): 863-869. 
496 Kristensen, K., Nielsen, A., Berg, C.W., Skaug, H., and Bell, B. 2015. TMB: automatic differ497 entiation and laplace approximation. arXiv preprint arXiv:1509.00660 .

498 Lacointe, A. 2000. Carbon allocation among tree organs: a review of basic processes and 499 representation in functional-structural tree models. Annals of Forest Science 57(5): 521$500 \quad 533$.

Lester, N.P., Shuter, B.J., and Abrams, P.A. 2004. Interpreting the von bertalanffy model of somatic growth in fishes: the cost of reproduction. Proceedings of the Royal Society of London. Series B: Biological Sciences 271(1548): 1625-1631.

Macdonald, P.D.M. and Pitcher, T.J. 1979. Age-groups from size-frequency data: a versatile and efficient method of analyzing distribution mixtures. Journal of the Fisheries Board of Canada 36(8): 987-1001.

McLachlan, G. and Peel, D. 2000. Finite Mixture Models. John Wiley \& Sons, Inc., New York, USA.

McLachlan, G. and Krishnan, T. 2008. The EM algorithm and extensions. John Wiley \& Sons, Inc., Hoboken, NJ, USA, 2nd edition.

Methot, R.D. 2000. Technical description of the stock synthesis assessment program. Technical report, NOAA.

Myers, R.A. and Hoenig, J.M. 1997. Direct estimates of gear selectivity from multiple tagging experiments. Canadian Journal of Fisheries and Aquatic Sciences 54(1): 1-9.

515 Nentwig, W. 2012. Ecophysiology of spiders. Springer Science \& Business Media.

Pardo, S.A., Cooper, A.B., and Dulvy, N.K. 2013. Avoiding fishy growth curves. Methods in Ecology and Evolution 4(4): 353-360. doi:10.1111/2041-210x.12020.

518 Parker, G.A. 2006. The evolution of sexual size dimorphism in fish. Journal of Fish Biology 519 41(sB): 1-20. 
520 Pauly, D. 1980. On the interrelationships between natural mortality, growth parameters, and 521 mean environmental temperature in 175 fish stocks. Journal du Conseil 39(2): 175-192.

Pearson, K. 1895. Contributions to the mathematical theory of evolution. ii. skew variation in homogeneous material. Philosophical Transactions of the Royal Society of London 186: $343-414$.

Ricker, W.E. 1975. Computation and interpretation of biological statistics of fish populations. Bulletin of the Fisheries Research Board of Canada 191: 382.

Robertson, T.B. 1923. The chemical basis of growth and senescence. JB Lippincott company, Philadelphia, USA.

Roff, D.A. 1982. Reproductive strategies in flatfish: a first synthesis. Can. J. Fish. Aquat. Sci. 39: $1686-1698$.

Roff, D. 1983. An allocation model of growth and reproduction in fish. . Can. J. Fish. Aquat. Sci. 40(9): 1395-1404.

Schnute, J. 1981. A versatile growth model with statistically stable parameters. Canadian Journal of Fisheries and Aquatic Sciences 38(9): 1128-1140.

Shumway, R.H. and Stoffer, D.S. 1982. An approach to time series smoothing and forecasting using the em algorithm. Journal of time series analysis 3(4): 253-264.

Starck, J.M. and Ricklefs, R.E. 1998. Avian growth and development: evolution within the altricial-precocial spectrum. 8. Oxford University Press.

Stearns, S.C. and Koella, J.C. 1986. The evolution of phenotypic plasticity in life-history traits: predictions of reaction norms for age and size at maturity. Evolution pp. 893-913.

Thorson, J.T., Stewart, I.J., Punt, A.E., and Jech, J.M. 2011. Accounting for fish shoals in single-and multi-species survey data using mixture distribution models. Canadian Journal of Fisheries and Aquatic Sciences 68(9): 1681-1693.

544 Ullman, J.B. and Bentler, P.M. 2003. Structural equation modeling. Wiley Online Library. 
545 Vitale, F., Svedäng, H., and Cardinale, M. 2006. Histological analysis invalidates macroscop546 ically determined maturity ogives of the kattegat cod (Gadus morhua) and suggests new 547 proxies for estimating maturity status of individual fish. ICES Journal of Marine Science $548 \quad$ 63(3): 485-492.

549 von Bertalanffy, L. 1938. A quantitative theory of organic growth. Human Biology 10: 181550213.

551 Walford, L.A. 1946. A new graphic method of describing the growth of animals. Biological $552 \quad$ Bulletin 90(2): 141-147.

553 Wang, P., Puterman, M., Cockburn, I., and Le, N. 1996. Mixed poisson regression models with 554 covariate dependent rates. Biometrics 52(2): 381-400.

555 Warner, R.R. 2012. Review of reproduction and sexuality in marine fishes: Patterns and pro556 cesses. Quarterly Review of Biology 87: 157.

557 Weisberg, S., Spangler, G., and Richmond, L.S. 2010. Mixed effects models for fish growth. 558 Canadian Journal of Fisheries and Aquatic Sciences 67(2): 269-277. doi:10.1139/F09-181.

559 Werner, E.E. 1986. Amphibian metamorphosis: Growth rate, predation risk, and the optimal 560 size at transformation. The American Naturalist 128(3): 319-341. 
Table 1: Root-median-square error by: parameter, method and whether maturation occurred early or late in the lifespan of the simulated species. Asymptotic and linear refer to the form of the simuated curves. Full, FMU and MIX refer to the complete knowledge, mature animals only and mixture model, respectively.

\begin{tabular}{|c|c|c|c|c|c|c|c|c|}
\hline & \multicolumn{2}{|c|}{$L_{\infty}$} & \multicolumn{2}{|c|}{$K$} & \multicolumn{2}{|c|}{$t_{0}$} & \multicolumn{2}{|c|}{$\sigma$} \\
\hline & Early & Late & Early & Late & Early & Late & Early & Late \\
\hline Asymptotic & & & & & & & & \\
\hline FULL & 0.899 & 0.901 & 0.019 & 0.020 & 0.060 & 0.061 & 0.007 & 0.007 \\
\hline FMU & 1.107 & 1.547 & 0.045 & 0.094 & 0.377 & 0.999 & 0.010 & 0.014 \\
\hline MIX & 0.912 & 0.987 & 0.022 & 0.028 & 0.076 & 0.092 & 0.009 & 0.011 \\
\hline Linear & & & & & & & & \\
\hline FULL & 2.013 & 2.067 & 0.008 & 0.008 & 0.045 & 0.045 & 0.007 & 0.007 \\
\hline FMU & 2.689 & 3.759 & 0.014 & 0.026 & 0.235 & 0.997 & 0.009 & 0.013 \\
\hline MIX & 2.090 & 2.348 & 0.008 & 0.010 & 0.055 & 0.063 & 0.008 & 0.010 \\
\hline
\end{tabular}




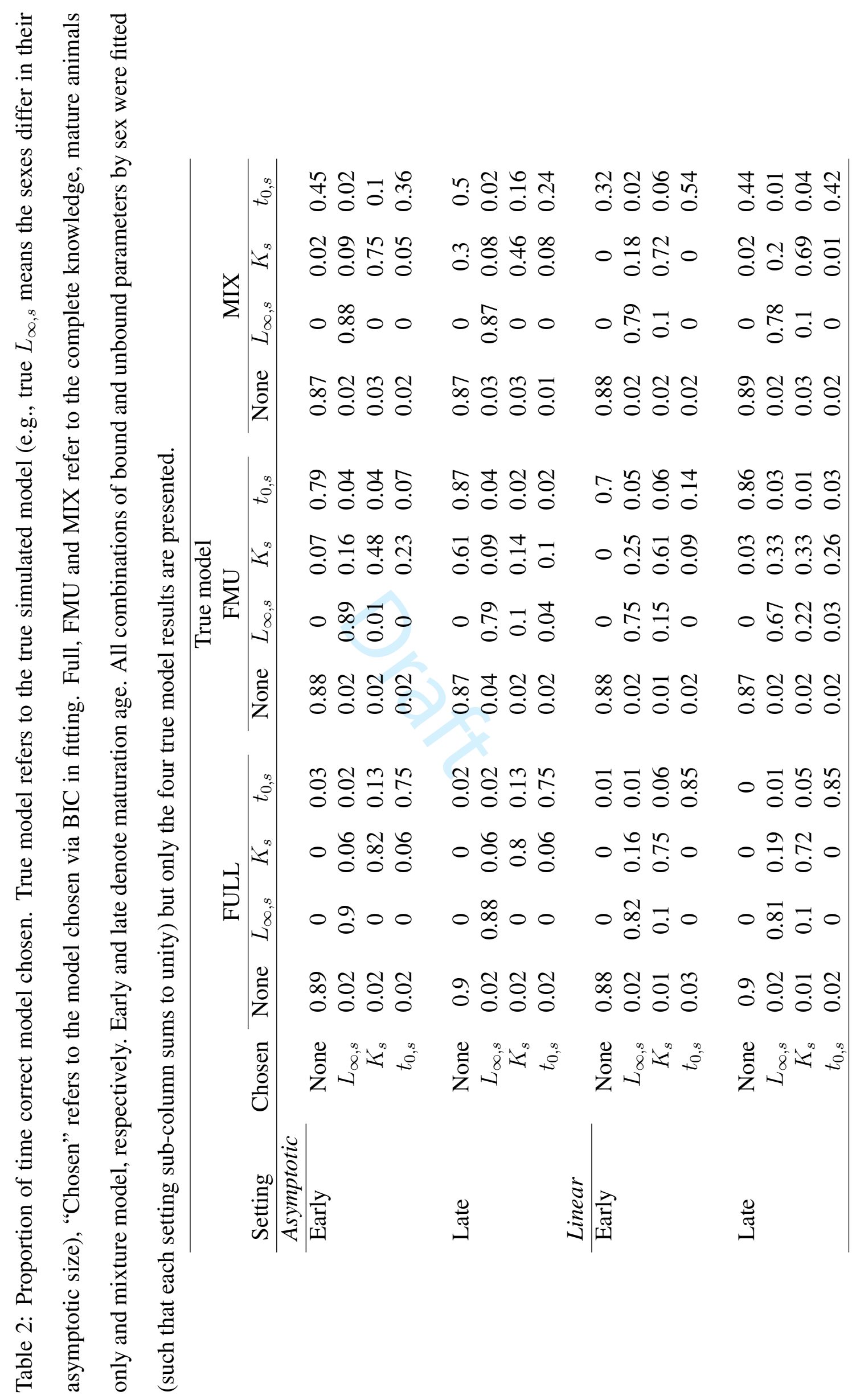


Table 3: Median maximum a posteriori classification rate across iterations. Asymptotic and linear refer to the form of the simuated curves. FULL, FMU and MIX refer to the complete knowledge, mature animals only and mixture model, respectively.

\begin{tabular}{|c|c|c|c|c|c|c|c|c|}
\hline & \multicolumn{2}{|c|}{$L_{\infty, s}$} & \multicolumn{2}{|c|}{$K_{s}$} & \multicolumn{2}{|c|}{$t_{0, s}$} & \multicolumn{2}{|c|}{ None } \\
\hline & Early & Late & Early & Late & Early & Late & Early & Late \\
\hline \multicolumn{9}{|l|}{ Asymptotic } \\
\hline FULL & 0.88 & 0.87 & 0.82 & 0.78 & 0.73 & 0.66 & 0.53 & 0.53 \\
\hline FMU & 0.77 & 0.64 & 0.69 & 0.52 & 0.48 & 0.48 & 0.47 & 0.48 \\
\hline MIX & 0.86 & 0.85 & 0.79 & 0.72 & 0.60 & 0.52 & 0.47 & 0.48 \\
\hline Linear & & & & & & & & \\
\hline FULL & 0.87 & 0.87 & 0.85 & 0.84 & 0.76 & 0.68 & 0.54 & 0.53 \\
\hline FMU & 0.79 & 0.66 & 0.77 & 0.64 & 0.51 & 0.48 & 0.48 & 0.48 \\
\hline MIX & 0.85 & 0.86 & 0.84 & 0.82 & 0.69 & 0.58 & 0.47 & 0.48 \\
\hline
\end{tabular}


Table 4: Best fitting (lowest AIC) von Bertalanffy parameter estimates by method for two deepwater lantern shark species Etmopterus spinax and Etmopterus pusillus. Full, FMU and MIX refer to the complete knowledge, mature animals only and mixture model, respectively. Standard errors are provided in parentheses.

\begin{tabular}{|c|c|c|c|c|c|c|c|}
\hline Species & Sex & Method & $\ln L_{\infty}$ & $\ln K$ & $\ln -t_{0}$ & $\ln \sigma$ & Class rate \\
\hline \multirow[t]{6}{*}{ E. spinax } & \multirow[t]{3}{*}{ Female } & FULL & $4.03(0.04)$ & $-2.16(0.07)$ & $0.7(0.05)$ & $0.65(0.03)$ & 0.65 \\
\hline & & FMU & $3.94(0.17)$ & $-2.41(0.55)$ & $1.89(0.44)$ & $0.2(0.04)$ & 0.08 \\
\hline & & MIX & $3.85(0.03)$ & $-1.74(0.07)$ & $0.42(0.08)$ & $0.41(0.03)$ & 0.35 \\
\hline & \multirow[t]{3}{*}{ Male } & FULL & $4.03(0.04)$ & $-2.34(0.06)$ & $0.92(0.04)$ & $0.44(0.04)$ & 0.46 \\
\hline & & FMU & $3.82(0.17)$ & $-2.41(0.55)$ & $1.89(0.44)$ & $0.2(0.04)$ & 1.00 \\
\hline & & MIX & $4.43(0.2)$ & $-2.97(0.27)$ & $1.14(0.08)$ & $0.41(0.03)$ & 0.80 \\
\hline \multirow[t]{6}{*}{ E. pusillus } & \multirow[t]{3}{*}{ Female } & FULL & $3.96(0.01)$ & $-1.92(0.05)$ & $0.92(0.06)$ & $0.42(0.03)$ & 0.33 \\
\hline & & FMU & $4.07(0.08)$ & $-2.65(0.29)$ & $2.35(0.23)$ & $0(0.06)$ & 0.12 \\
\hline & & MIX & $3.94(0.01)$ & $-1.77(0.04)$ & $0.88(0.05)$ & $-0.19(0.11)$ & 0.21 \\
\hline & \multirow[t]{3}{*}{ Male } & FULL & $3.94(0.02)$ & $-1.92(0.05)$ & $0.92(0.06)$ & $0.42(0.03)$ & 0.84 \\
\hline & & FMU & $4.07(0.08)$ & $-2.77(0.27)$ & $2.35(0.23)$ & $0(0.06)$ & 0.98 \\
\hline & & MIX & $3.94(0.01)$ & $-1.91(0.03)$ & $0.88(0.05)$ & $0.34(0.04)$ & 0.91 \\
\hline
\end{tabular}




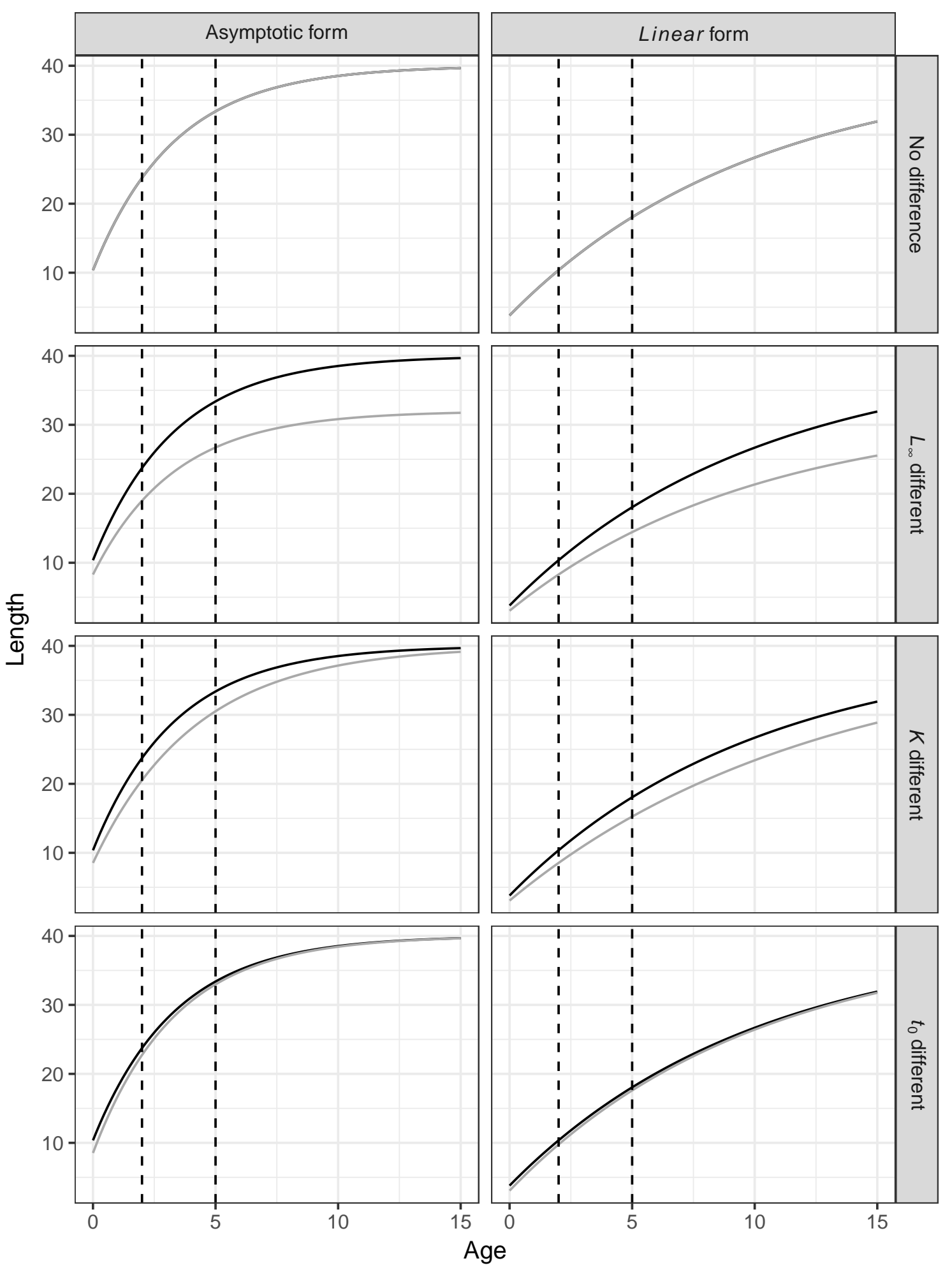

Figure 1: Simulation framework illustration. Asymptotic and linear (weakly asymptotic) refer to the form of the simulated curves. Rows denote parameter differences between females and males. Female and male mean curves are shown in black and grey, respectively. Dashed vertical lines denote early and late maturation ages. 


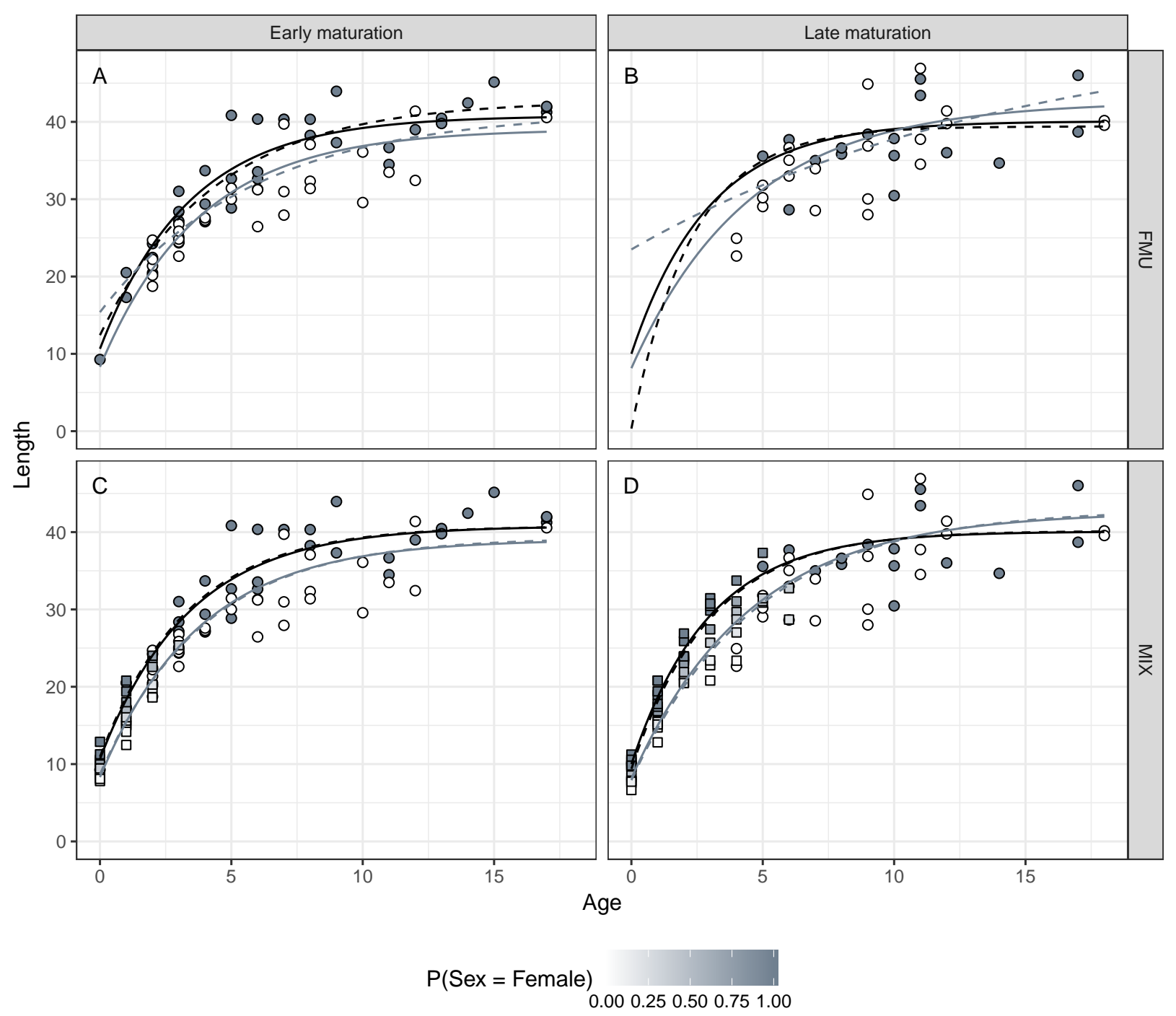

Figure 2: Example simulations for an early maturing (left column) and late maturing (right column) population. FMU and MIX refer to the mature animals only and mixture models, respectively. Full data curves are displayed as solid lines (females black, males grey). Estimated FMU and MIX curves are shown as short-dashed and long-dashed curves, respectively. Known-sex individuals are shown as circles and unknown-sex individuals as squares. The fill colour of the points denotes the probability of the individual being female (from the MIX model for unknowns). 


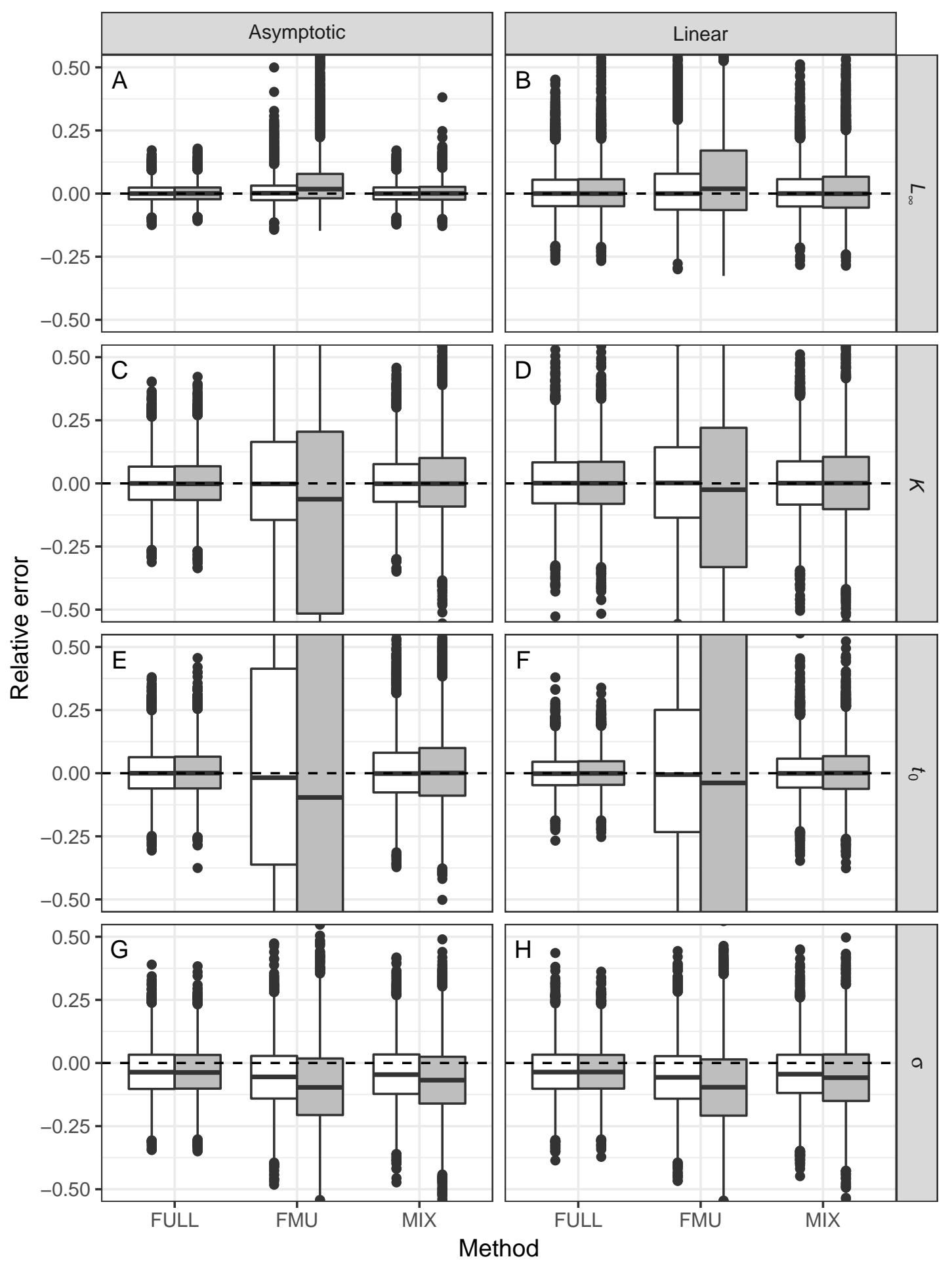

Figure 3: Boxplots displaying the distribution of the relative error by: parameter, method, and whether maturation occurred early (white) or late (grey) in the lifespan of the simulated species. Boxes correspond to the 25th and 75th percentiles, whiskers extend to the closest points to one and a half times the interquartile range. Full, FMU and MIX refer to the complete knowledge, mature animals only and mixture model, respectively. Asymptotic and linear refer to the form of the simuated curves (Figure 1). Y-axis range is restricted to facilitate comparison of most distributions. 

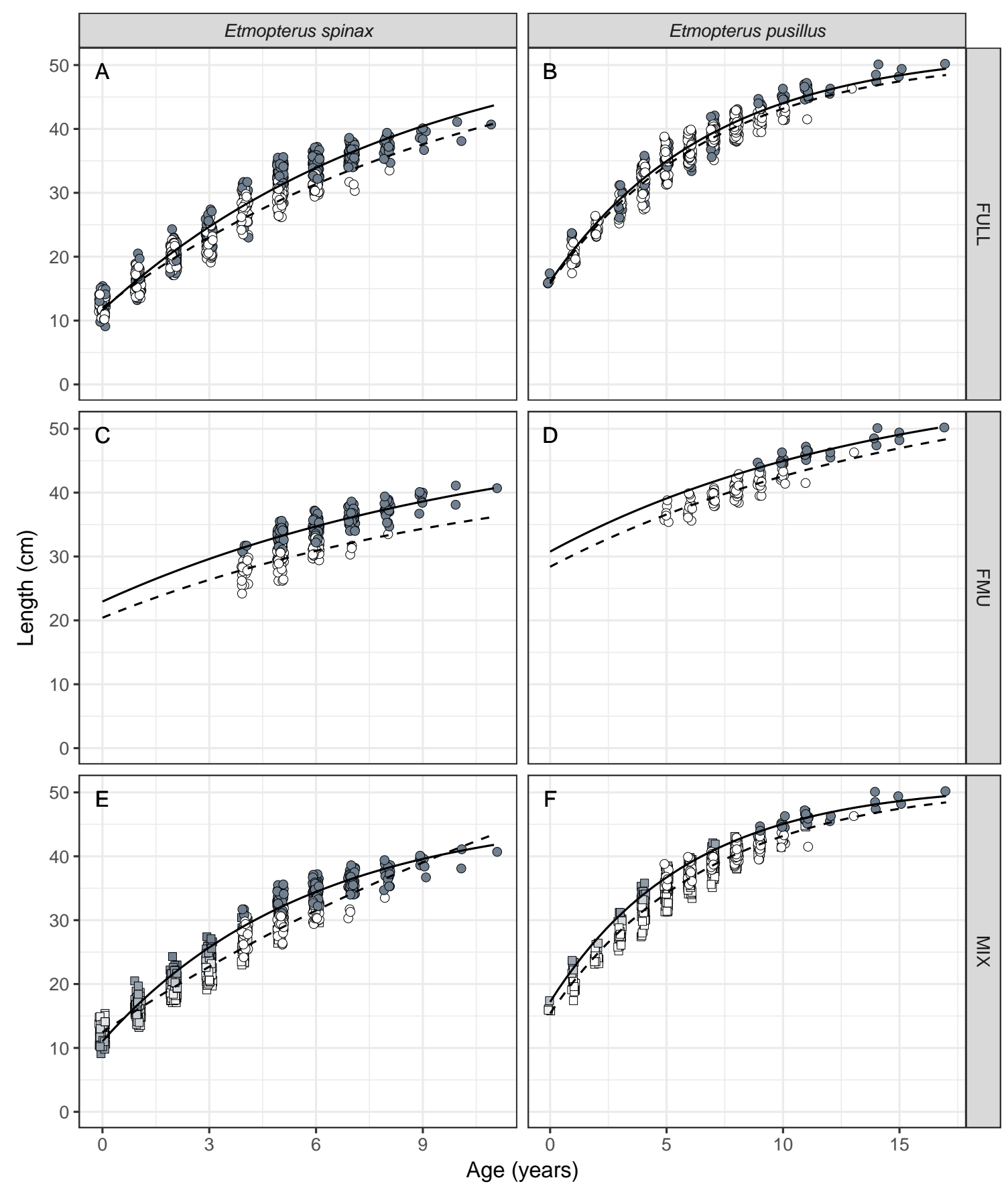

$$
\mathrm{P}(\text { Sex }=\text { Female })
$$

0.000 .250 .500 .751 .00

Figure 4: Best fitting (lowest BIC) von Bertalanffy fits to age-length data from two deepwater lantern sharks species Etmopterus spinax and Etmopterus pusillus. Full, FMU and MIX refer to the complete knowledge, mature animals only and mixture model, respectively. Estimated curves for females and males are shown as solid and dashed lines, respectively. Known-sex individuals are shown as circles and unknown-sex individuals as squares. The fill colour of the triangles in the MIX model case denote the probability of the individual being female. Age values are jittered for visualization. 